\title{
LA GERENCIA DEL MARKETING EDUCATIVO EN EL CONTEXTO \\ UNIVERSITARIO DEL ESTADO YARACUY
}

Hamlet Trejo y Mireya Valdez.

(PP. 4-26)
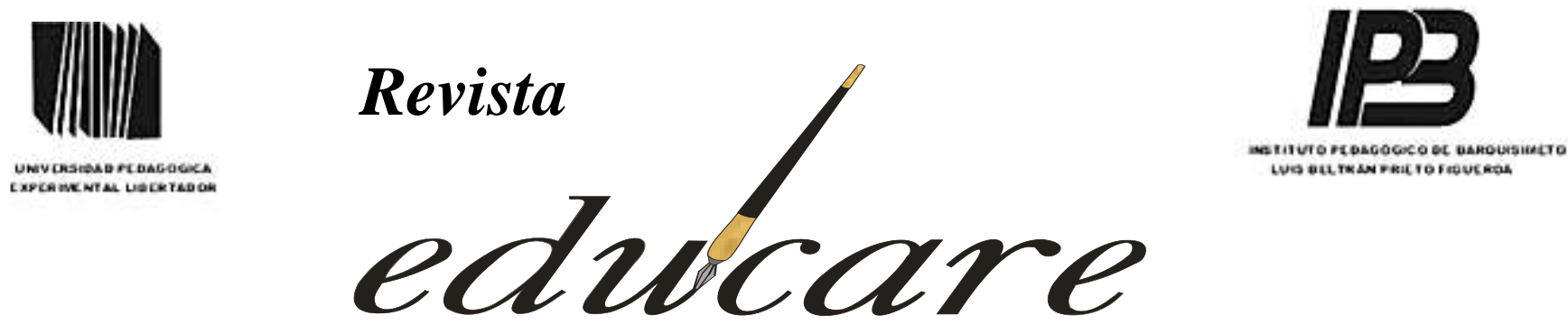

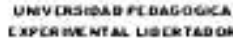

Órgano Divulgativo de la Subdirección de Investigación y Postgrado del Instituto Pedagógico de Barquisimeto "Luis Beltrán Prieto

Figueroa”

Barquisimeto estado Lara

EDICIÓN XX ANIVERSARIO

Volumen 21 № $2 \quad$ Mayo - Agosto 2017
LA GERENCIA DEL MARKETING EDUCATIVO EN EL CONTEXTO
UNIVERSITARIO DEL ESTADO YARACUY
EDUCATIONAL MARKETING MANAGEMENT IN THE UNIVERSITY ENVIRONMENT
FROM YARACUY STATE
I S S : $244-7296$

\begin{abstract}
Autor
Mireya Valdez

Colegio Universitario de Administración y Mercadeo Extensión San Felipe

Hamlet Trejo

Universidad Centro Occidental Lisandro Alvarado (UCLA)
\end{abstract}




\title{
LA GERENCIA DEL MARKETING EDUCATIVO EN EL CONTEXTO \\ UNIVERSITARIO DEL ESTADO YARACUY
}

Hamlet Trejo y Mireya Valdez

(PP. 4-26)

\section{$\underline{\text { Investigación }}$}

Recibido: 01-12-2016

\section{RESUMEN}

En la actualidad las universidades deben realizar su misión en ambientes de incertidumbres, por esta razón se plantea que la gerencia en aras de lograr la consolidación de la oferta académica, debe desarrollar estrategias, como el marketing como una herramienta para la captación de estudiantes. Desde esta perspectiva, se planteó una tesis doctoral cuyo propósito fue construir una aproximación teórica de la gerencia del marketing educativo en el contexto universitario del estado Yaracuy. Para la investigación se seleccionó el enfoque cualitativo, con una postura ontológica a través de la teoría de las realidades múltiples. Epistemológicamente el estudio se basó en la intersubjetividad. Se empleó la fenomenología y la hermenéutica como método puesto que contribuyen a develar los significados intersubjetivos que tienen los actores sociales sobre el marketing educativo. La técnica usada fue la entrevista a profundidad a nueve (9) actores sociales, pertenecientes a tres instituciones de educación universitarias del estado Yaracuy. Se utilizó la triangulación de fuentes para develar los hallazgos, entre los cuales destaca, que la captación de estudiantes resulta favorecida por la imagen institucional, la calidad educativa y la proyección de la universidad en la comunidad.

Descriptores: Gerencia Universitaria, Marketing Educativo

\author{
Hamlet Trejo* \\ Colegio Universitario de Administración y Mercadeo \\ Extensión San Felipe \\ Mireya Valdez ${ }^{* *}$ \\ Universidad Centro Occidental Lisandro Alvarado \\ (UCLA)
}

Aceptado:15-03-2017

\begin{abstract}
Nowadays, the universities must achieve their mission in environments of uncertainty; in this sense, it is proposed that the management targeting the consolidation of the academic offer must develop strategies like marketing as a tool for recruiting students. From this perspective, it was proposed a doctoral dissertation pursuing the goal of building a theoretical approach of educational marketing management in the higher education context in Yaracuy. For the study, the qualitative approach was selected, with an ontological view towards the theory of multiple realities. The study was epistemologically based on the intersubjectivity. Phenomenology and hermeneutics were used as methods because they contribute to show the intersubjective meanings that social actors possess on educational marketing. The selected technique for gathering information was the in-deep interview to nine (9) social actors belonging to three universities from Yaracuy. Sources triangulation was used to unveil the findings, among which highlights that students capacitation is favored by the institutional image, educational quality and image of the university to the community.
\end{abstract}

Keywords: University management, educational marketing.

Doctor en Gerencia, Magister en Gerencia Educacional y Licenciado en Administración de Empresas. Docente del Colegio Universitario de Administración y Mercadeo extensión San Felipe estado Yaracuy hamletre@ gmail.com

*** Doctora en Ciencias Humanas, Magister en Educación. Mención Investigación Educativa, Especialista en Metodología de la Investigación, Licenciada en Educación, Directora del Programa de Agroindustrial del Decanato de Agronomía de la Universidad Centro Occidental Lisandro Alvarado mireyavaldez@ucla.edu.ve 


\section{LA GERENCIA DEL MARKETING EDUCATIVO EN EL CONTEXTO \\ UNIVERSITARIO DEL ESTADO YARACUY}

Hamlet Trejo y Mireya Valdez.

(PP. 4-26)

\section{INTRODUCCIÓN}

El tercer milenio representa una época de constantes y vertiginosos cambios, que afectan la vida cotidiana de todos los habitantes de este siglo y de los que tampoco escapan las organizaciones. Es así como, una gerencia organizacional eficaz en los tiempos actuales implica no sólo disponer de conocimientos y habilidades, sino manejar herramientas innovadoras, poseer una visión estratégica que permita identificar el rumbo que debe seguir la institución, tener una serie de principios, entender y valorar al talento humano que la integra como su elemento fundamental.

En el caso de las Instituciones de Educación Universitaria (IEU) en este escenario y tal como lo plantean Muro y Picón (2005), los cambios experimentados han sido mayores y más continuos que la adopción de nuevos enfoques y modelos, por lo que existe un marcado déficit de entendimiento, interpretación y comprensión por parte de sus integrantes (tanto de la gerencia como el resto del personal) de las nuevas realidades institucionales.

Contextualizando en el escenario nacional, Rivero y Goyo (2012), señalan que las universidades venezolanas deben transformarse en organizaciones inteligentes, donde los cambios y su proyección en el entorno en el cual se desarrollan, les permita afrontar realidades complejas para así poder mantener su vigencia, por lo que la gerencia universitaria requiere movilidad y una dinámica constante dentro del tiempo y espacio, a objeto de cumplir con la misión para las cuales están llamadas las IEU, con la búsqueda de la eficiencia y de una imagen de excelencia y productividad.

Sobre el particular Malpica y Rossel (2010), plantean que esta gerencia debe retomar los valores éticos, la moral, las habilidades, destrezas, compromisos, sentidos de pertenencia, meritocracia, trabajo en equipo, estrategias, motivaciones, la inclusión y distribución de responsabilidades, entre otros, para que del seno de las IEU se inicie una transformación.

De lo planteado hasta aquí, se enfatiza la idea de que las universidades y demás instituciones de educación universitaria deben renovarse a la luz de los tiempos actuales, lo que constituye no sólo la actualización de programas y currículo, sino el acercamiento a las comunidades, empresas, gobiernos y demás fuerzas vivas de la sociedad para convertirse en verdaderos dinamizadores del progreso económico, social y ambiental que requiere el país. 


\section{LA GERENCIA DEL MARKETING EDUCATIVO EN EL CONTEXTO \\ UNIVERSITARIO DEL ESTADO YARACUY}

Hamlet Trejo y Mireya Valdez.

(PP. 4-26)

Dentro de este orden de ideas, se reconoce la contribución fundamental de las entidades universitarias en la construcción de una visión de país y el funcionamiento de sus distintos órganos, por lo que la responsabilidad de la gerencia se expresa, no sólo con la organización que representa sino con toda la sociedad, lo que implica comprometerse con el éxito de la institución a través de su permanencia en el tiempo y la innovación constante en productos y servicios.

Desde esta perspectiva, se estima que el aprovechamiento de las oportunidades es un componente clave para el éxito de cualquier organización, incluyendo las educativas, pero implica que su gerencia debe estar preparada, manteniéndose atenta a las tendencias del mercado, preferencias de consumidores y en mucho de los casos crear las circunstancias propicias para diseñar una poderosa ventaja competitiva.

Cabe destacar, que conocer precisamente esas demandas y expectativas de los grupos sociales que constituyen el mercado meta de las organizaciones, es esencialmente un área de especial estudio para la mercadotecnia o marketing, que adicionalmente implica lo que son las necesidades y deseos de quienes forman los clientes actuales y potenciales de las entidades organizativas.

En lo esencial, Sainz (2010) indica que el marketing se trata de una orientación empresarial que reconoce que el éxito de la organización sólo es sostenible si esta se concibe para satisfacer las necesidades actuales y futuras de los clientes, consumidores o usuarios de una forma más eficaz que los competidores, lo que aumenta la rentabilidad corporativa.

Por su parte, conviene recordar que las universidades, institutos y colegios universitarios son, organizaciones académicas que ofrecen un servicio, que está dirigido a un mercado meta formado por estudiantes actuales y potenciales que constituyen los usuarios, o de acuerdo con los enfoques de calidad educativa, los clientes externos, quienes poseen sus expectativas, formadas por necesidades, deseos $\mathrm{y}$ demandas en torno a lo que las instituciones pueden ofrecerles.

Como todas las instituciones actuales, éstas se desenvuelven en un entorno dinámico y de incertidumbre, que exige la renovación y aprendizaje permanente, signadas por la presencia de competidores o entes sociales que ofrecen servicios académicos similares, los 


\section{LA GERENCIA DEL MARKETING EDUCATIVO EN EL CONTEXTO \\ UNIVERSITARIO DEL ESTADO YARACUY}

Hamlet Trejo y Mireya Valdez.

(PP. 4-26)

cuales están igualmente interesados en lograr la atención de los estudiantes para incorporarlos a sus procesos, al tiempo que cumplen con su misión educativa y tratan de mantenerse en el camino que los llevará a la realización de la visión organizacional.

Es así que estas condiciones obligan a la gerencia universitaria, a hacer uso de algunas herramientas estratégicas, tales como el marketing, aunque adaptado a sus necesidades y bajo unos condicionamientos éticos particulares, ya que la educación se trata de un servicio que al mismo tiempo es un derecho consagrado constitucionalmente, y es por lo tanto una actividad regulada por el Estado, en el caso venezolano a través del Ministerio del Poder Popular para la Educación Universitaria la Ciencia y la Tecnología (MPPEUCT).

Sobre este particular, a juicio de Saldaña (2013), los conceptos del marketing se pueden aplicar a los servicios de educación, investigación y difusión de la cultura, así como en la promoción de centros de enseñanzas que persiguen modificar la realidad y ser agentes de la transformación social hacia la obtención de calidad de vida de los ciudadanos.

Desde este contexto, se considera que el marketing educativo es además un elemento de gran relevancia para las IEU, pues generalmente existe entre ellas la competencia, no sólo entre las de carácter privado, sino también en las públicas, especialmente cuando en los últimos años, de acuerdo con la Misión Alma Mater (2012), han aumentado la cantidad de organizaciones educativas públicas que ofrecen las mismas carreras o similares, a las que se accede de manera gratuita.

A partir de los argumentos anteriores se considera que, las organizaciones universitarias precisan desenvolverse y adaptarse a entornos cambiantes y complejos, la gerencia cumple un rol fundamental no sólo en las funciones administrativas que le son propias, sino también en la imagen, proyección y aportes que realiza la institución al país y a la sociedad, además de la obtención de una o más ventajas competitivas que le permitan alcanzar el éxito, por consiguiente el uso del marketing educativo parece pertinente.

Sobre este tema se realizó una entrevista a un experto en marketing, quien manifestó lo siguiente:

Las universidades privadas principalmente hacen uso del marketing para llegar a un mercado meta que está formado por los alumnos y los padres que son quienes pagan, para ofrecer un producto intangible como lo es la educación. En Venezuela dada la situación económica poco se practica pero a través de la televisión por cable vemos 


\section{LA GERENCIA DEL MARKETING EDUCATIVO EN EL CONTEXTO \\ UNIVERSITARIO DEL ESTADO YARACUY}

Hamlet Trejo y Mireya Valdez.

(PP. 4-26)

como universidades internacionales hacen publicidad para captar estudiantes de otros países...El marketing que utilizan las universidades privadas, lo puede utilizar tanto a nivel de postgrado como a nivel de pregrado, y la idea es que los estudiantes de pregrado queden enganchados con la universidad y hagan la recompra, es decir cursen su estudios de postgrado en la misma universidad.

De los aportes de este especialista destaca, que el marketing es una herramienta de gran importancia para las IEU que ofrecen postgrados para la captación de estudiantes, por lo que deberían crear relaciones de valor con los egresados de pregrado, a través de la satisfacción de la calidad del producto intangible o servicio educativo ofrecido, para que estos realicen la prosecución académica (diplomados, especializaciones, maestrías o doctorados) en la misma institución.

Así mismo, cabe señalar que en párrafos anteriores se ha destacado la calidad que debe tener el servicio educativo que brindan las IEU, al igual que la idoneidad del talento humano, conformado fundamentalmente en este caso por el personal docente, y los valores de la organización. Sobre este particular, un experto en marketing educativo expresó su opinión en los siguientes términos:

En el caso de las universidades hay dos procesos captación y retención de estudiantes. Para la captación hay que ofrecer un buen producto académico, la retención es porque hay que crear fidelización, que no haya deserción...por eso yo te hablaba del producto académico, si el producto es malo ni la mejor publicidad lo vende, por lo tanto es importante ser ético...¿qué pasa si uno no es ético? y promete computadores que no existen o hay profesores que no tienen curriculum, entonces el alumno se da cuenta y por otro lado captarás mucho pero te durará solo un año y te va a provocar deserción. Si uno no es ético se provoca deserción, entonces empiezan a irse porque se dan cuenta de que "me prometieron equipos que no había, el profesorado es de mala calidad, programas anticuados, blablablá", entonces...si el producto académico no es correcto se va a producir la deserción, o sea vas a captar mucho y por otro lado en un año se te van a ir los alumnos también, por lo tanto hay que decir la verdad, pero una verdad bien contada, es como uno cuenta las cosas, en base a lo que hay, pero sin mentiras, hay instituciones que mienten, sobre todo que disfrazan las fotos, disfrazan los vídeos, disfrazan un montón de cosas y en el fondo el alumno cuando llega se da cuenta, y uno de los mayores problemas que hay es que, es la metodología de la enseñanza, están enseñando a la generación zeta, nacidos después del año noventa y cuatro, por lo tanto, los profesores deben adaptar su metodología porque eso también provoca deserción, si no están actualizados con lo que, con la manera de enseñar, ¿qué están haciendo? 


\section{LA GERENCIA DEL MARKETING EDUCATIVO EN EL CONTEXTO \\ UNIVERSITARIO DEL ESTADO YARACUY}

Hamlet Trejo y Mireya Valdez.

(PP. 4-26)

Las afirmaciones anteriores, enfatizan los aspectos éticos que deben orientar al marketing educativo en el contexto universitario, donde la oferta del producto debe estar acorde no sólo con las expectativas de los estudiantes, sino además con lo que la institución realmente puede ofrecer, de igual forma se destaca la importancia de cualquier institución académica de contar con docentes actualizados, que utilicen las nuevas tecnologías y que desempeñen sus funciones de manera profesional.

Igualmente, como una primera aproximación al fenómeno de estudio en el escenario de la investigación, se realizó una entrevista no estructurada a un informante clave, quien se desempeña como gerente de una IEU del estado Yaracuy, el cual expresó lo siguiente sobre lo que es el marketing educativo y lo realizado específicamente en esa institución:

La estrategia que usamos, como se trata de una oferta educativa de gestión privada, tenemos que garantizar, pues una mayor divulgación de lo que es la oferta académica, para que llegue a la población meta, un producto que en este caso son los servicios educativos y efectivamente pues la institución tiene algunas estrategias que son diseñadas desde el nivel central que pautan publicidad en distintos medios, específicamente en prensa, ellos diseñan una plantilla única pues de lo que será el aviso publicitario que publican en el periódico de mayor circulación en las sedes, en las regiones donde están ubicadas las sedes, valga decir...Hay un coordinador nacional de publicidad y mercadeo y él da esas estrategias, eso no exime que a nivel de sedes por iniciativa del director y administrador como efectivamente lo hemos diseñado algunas estrategias como ya en función de las redes sociales.

Del argumento anterior, se enfatiza que las IEU venezolanas resultan propicias para el estudio del marketing educativo por lo que se seleccionaron el Colegio Universitario de Administración y Mercadeo (CUAM), el Instituto Universitario de Tecnología Antonio José de Sucre (IUTAJS) y la Universidad Politécnica Territorial de Yaracuy Arístides Bastidas (UPTYAB), organizaciones ubicadas en el estado Yaracuy, como contexto para la presente investigación.

\section{PROPÓSITOS DE LA INVESTIGACIÓN}

Develar el significado intersubjetivo que los actores sociales le otorgan al marketing educativo en el contexto universitario del estado Yaracuy. 


\section{LA GERENCIA DEL MARKETING EDUCATIVO EN EL CONTEXTO \\ UNIVERSITARIO DEL ESTADO YARACUY}

Hamlet Trejo y Mireya Valdez.

(PP. 4-26)

Interpretar los elementos significativos que surjan, como categorías del discurso de los actores sociales en relación al marketing educativo en el contexto universitario del estado Yaracuy.

Integrar la conexión de los significados sociales emergentes del marketing educativo en el contexto universitario del estado Yaracuy, sobre la base de los hallazgos categoriales.

Construir una aproximación teórica a la gerencia del marketing educativo en el contexto universitario del estado Yaracuy, a partir de las voces de sus actores sociales.

\section{ARGUMENTACIÓN TEÓRICA}

\section{Gerencia universitaria}

Como se ha mencionado con anterioridad, el siglo XXI se destaca como una época de constantes y vertiginosos cambios que afectan a las instituciones, por lo cual están obligadas a renovarse continuamente. De esta realidad no escapan los centros de educación universitaria, que según Didriksson (2005), por ser instituciones de gran trascendencia para fines de desarrollo económico, cultural y social, las expectativas que se ciernen sobre estas, están provocando presiones sin límites, la redefinición de políticas y planes, la frecuencia de programas y alternativas en la búsqueda de nuevos modelos de organización.

Esta visión es compartida por Knight, (2005) al argumentar que la educación universitaria está cambiando por numerosas razones, tales como el desarrollo de servicios avanzados de comunicación y tecnología, la mayor movilidad de mano de obra internacional, el énfasis en la economía de mercado y liberación comercial, el enfoque en la sociedad del conocimiento, el aumento de la inversión privada y menor apoyo público para la educación, así como también la importancia cada vez mayor del aprendizaje permanente.

Desde esta perspectiva, algunos de los elementos señalados por Knight están presentes en la realidad venezolana mientras que en otros casos se marcha en sentido contrario, pero produciendo igualmente consecuencias en las IEU que también deben ser analizadas. De esta forma, el contexto nacional de los años recientes no se caracteriza por el aumento de la inversión privada, sino que el poder ejecutivo ha creado nuevas universidades, y la 


\section{LA GERENCIA DEL MARKETING EDUCATIVO EN EL CONTEXTO \\ UNIVERSITARIO DEL ESTADO YARACUY}

Hamlet Trejo y Mireya Valdez.

(PP. 4-26)

liberación económica del mercado es una política contraria a las implementadas por un Estado controlador y regulador de dichas actividades.

Sin embargo, se consideran relevantes algunos impactos que inciden en la sociedad del conocimiento, tales como, el desarrollo de las tecnologías de información y comunicación, el adelanto de nuevos planes y programas educativos, el acceso creciente de la población a contenidos internacionales a través de las redes informáticas, el aumento de la demanda de los servicios académicos tradicionales, a los que también se suman las necesidades de formación permanente de profesionales.

De esta manera, se estima como de trascendental importancia las funciones de una gerencia universitaria, que está obligada a conducir de manera exitosa los centros de enseñanzas y que trata de transitar una ruta compleja y difícil, donde se deben sopesar muchos elementos, tanto del entorno como internos, además de considerar un conjunto de variados escenarios.

En este orden de ideas y en un primer intento para la aproximación de un concepto de gerencia universitaria, Taplero y López (2006), aportan la definición de gestión escolar, la cual se refiere a las formas de abordar los desarrollos académico-administrativos de los centros educativos, noción que se encuentra sujeta a futuras resemantizaciones, con objeto de contrastar su tradición constituida, con el campo de tensión de las reformas educativas.

Estos autores complementan la idea al destacar que mientras las reformas en sentido descendente, optan por la heterorregulación, es decir, el desarrollo heterónomo, formal y burocratizado de lo normado, con disminución extrema de estímulos para el desempeño docente, las reformas educativas en sentido ascendente, hacen de la norma una fuente de autonomía para gestionar en forma proactiva el desarrollo escolar y, por tanto, estimulan la excelencia del desempeño profesional de la docencia por convertirse en artífices de la modernización institucional.

Así se infiere de lo anterior, que ya la definición de gerencia, incluye a la gestión como uno de sus elementos, contempla por si sola implementar cambios, que la flexibilidad en un ambiente laboral de libertad es una característica importante o deseable, además de que la participación en los procesos por parte de los profesores es una condición indispensable. 


\section{LA GERENCIA DEL MARKETING EDUCATIVO EN EL CONTEXTO \\ UNIVERSITARIO DEL ESTADO YARACUY}

Hamlet Trejo y Mireya Valdez.

(PP. 4-26)

En consecuencia, una definición de gerencia universitaria puede darse en los siguientes términos: Conjunto de habilidades y técnicas concebidas para lograr proporcionar a los centros de enseñanza universitaria, a través de la cooperación de todo el talento humano que los constituye, de un desarrollo académico-administrativo armónico con el entorno, que responda a las necesidades económicas, tecnológicas, científicas, culturales y sociales de una población.

Es así como se estima, que la gerencia universitaria para diseñar estrategias de manera eficiente, precisa tanto del conocimiento de las preferencias de los estudiantes actuales y potenciales (clientes externos), como de las motivaciones de los docentes y personal de apoyo (clientes internos), a la par de fomentar la calidad en los procesos de formación y todas las labores que desarrolle, para consolidar relaciones de valor y crear una ventaja competitiva.

En estas circunstancias resulta útil para las instituciones universitarias el implemento del marketing, ya que a juicio de Pérez (2004), este es una orientación donde la detección de las necesidades del cliente, constituyen el punto inicial para crear una oferta que genere ingresos, cubra costos y produzca rentabilidad en entornos competitivos de mercado. Este tópico será abordado a continuación.

\section{Marketing Educativo}

Aunque la mercadotecnia nació en el siglo XX como una herramienta empresarial para la mercantilización de productos, en la actualidad ha desbordado su ámbito inicial para ser empleada por cualquier tipo de organización lucrativa o no, con la finalidad de posicionar bienes tangibles o intangibles tales como servicios o ideas, de modo que resulta legítimo también su manejo como estrategia de captación de estudiantes por parte de los centros de enseñanza.

Desde esta perspectiva expresa Saldaña (ob. cit.), que existen diferencias entre vender productos y prestar servicios de educación, pero en ambos casos se trata de un intercambio de valor que no necesariamente se expresa en términos monetarios, sino que también involucra la percepción del estudiante y los grupos sociales acerca de la calidad de la oferta académica y otras actividades relacionadas. 


\section{LA GERENCIA DEL MARKETING EDUCATIVO EN EL CONTEXTO \\ UNIVERSITARIO DEL ESTADO YARACUY}

Hamlet Trejo y Mireya Valdez.

(PP. 4-26)

Asimismo explica esta autora que, la educación es un servicio porque es intangible, inseparable de la institución que la ofrece, genera resultados variables dependiendo de las condiciones al momento de prestarlo y las personas involucradas, además se produce en la presencia y con la colaboración del usuario y sobre todo establece una relación prolongada entre el individuo y la institución.

Por su parte, Manes (2008) argumenta que, el marketing educativo consiste en el proceso de investigación de las necesidades sociales, para desarrollar servicios educativos con la idea de satisfacerlas, de acuerdo con un valor percibido, distribuido en un tiempo y lugar, y éticamente promocionado en aras de generar un beneficio entre individuos y las organizaciones.

Mientras que de acuerdo con Bustos (2010), las estrategias de marketing educacional permiten desarrollar un posicionamiento dinámico de adaptación a la oferta y la demanda, ofreciendo servicios formativos que satisfacen las necesidades de los usuarios de acuerdo a los valores de cada institución, comunicando la calidad de educación de una manera éticamente coherente.

De acuerdo con las disertaciones anteriores, se interpreta que el marketing educativo, constituye una compleja herramienta estratégica, que coadyuva a la gerencia universitaria en la búsqueda de una o más ventajas competitivas dentro del mercado al que pertenece, al proporcionar elementos para potenciar a la institución a través de diferentes actividades, que encausadas por los valores organizacionales, le permitirá satisfacer un conjunto de necesidades sociales a través de la calidad.

\section{ASPECTOS METODOLÓGICOS}

\section{Naturaleza de la Investigación}

Se hizo un abordaje desde el paradigma interpretativo haciendo uso de los métodos y técnicas propias de la investigación cualitativa, específicamente de la fenomenología apoyado en las bondades de la hermenéutica.

Ontológicamente el estudio se realizó desde la perspectiva de las realidades múltiples, pues se precisa del significado que tiene el fenómeno para los actores sociales, quienes se 


\section{LA GERENCIA DEL MARKETING EDUCATIVO EN EL CONTEXTO \\ UNIVERSITARIO DEL ESTADO YARACUY}

Hamlet Trejo y Mireya Valdez.

(PP. 4-26)

expresan influenciados por su entorno social, cultural y sobre las bases de sus experiencias. Desde esta perspectiva, se distingue al marketing educativo como una realidad construida socialmente, producto de las interacciones sociales y culturales de los grupos humanos que hacen vida en estas instituciones, quienes le confieren características específicas, e interrelacionadas.

Adicionalmente, por razones de interés investigativo se consideró al fenómeno de manera global, lo que se supone proporciona una mayor y mejor comprensión del estudio, en aras de tener una visión holística, pero sin alejarse de quienes son los elementos que interactúan para proporcionarle su carácter de totalidad.

Desde el punto de vista epistémico,el estudio se basa en la interpretación intersubjetiva, pues resulta del producto de los actores sociales que vivencian una realidad socialmente compartida en una institución de educación universitaria, por lo que igualmente se sustenta en el construccionismo social o epistemología construccionista.

\section{Métodos}

Se utilizó la fenomenología que según Sandín (2003), es el estudio de las estructuras de la conciencia que posibilitan su relación con los objetos. Este estudio requiere la reflexión sobre el contenido de la mente, excluyendo todo lo demás. Se seleccionó este método, ya que se estima que contribuye a develar los significados intersubjetivos que tienen los actores sociales el marketing educativo, por ello el investigador y a la vez integrante de una de las instituciones del estudio, precisó situar entre paréntesis el mundo objetivo, para reflexionar sobre lo que al respecto constituyen deseos, recuerdos y sentimientos para percibir los contenidos que representan.

El método fenomenológico fue complementado con la hermenéutica, que para Heidegger (1999), se encarga de interpretar lo que lleva al encuentro de la visión, y al concepto de manejo de la facticidad. Este método permitió interpretar los elementos significativos emergentes como categorías del discurso de los actores sociales. 


\section{LA GERENCIA DEL MARKETING EDUCATIVO EN EL CONTEXTO \\ UNIVERSITARIO DEL ESTADO YARACUY \\ Hamlet Trejo y Mireya Valdez. \\ (PP. 4-26)}

\section{Técnica de Recolección de Información}

La recolección de información se hizo a través de entrevistas a profundidad de carácter individual.

\section{Actores Sociales}

Tres (3) estudiantes, tres (3) docentes y tres (3) gerentes. Pertenecientes al escenario de estudio formado por el IUTAJS, el CUAM y la UPTYAB, que constituyen IEU del estado Yaracuy.

\section{Técnicas de Interpretación de la Información Categorización y Triangulación}

Se transcribieron las entrevistas conjuntamente con los campos categorías, subcategorías y código, mientras la organización de la información se efectuó a través de los elementos significativos de los discursos de los actores sociales, la triangulación e interpretación junto con la integración de los significados sociales emergentes, se efectuó por categoría.

\section{Validez y Credibilidad}

Para esta investigación en aras de lograr la validez y credibilidad de los hallazgos, se empleó la triangulación de fuentes a través de entrevistas a diferentes actores sociales que desempeñan roles similares en tres instituciones universitarias diferentes, y como apoyo de esta técnica se utilizó como recursos las grabaciones de audio.

\section{PRESENTACIÓN DE LOS HALLAZGOS}

Producto del proceso de categorización se derivaron cinco (5) categorías nucleares, a saber: Mercado Educativo Universitario, Cliente Externo Estudiante; Cliente Interno Docente, Entorno Institucional y Sistema Organizacional, y veinte (20) subcategorías.

\section{Categoría: Mercado Educativo Universitario}

Las subcategorías que la forman aparecen en la imagen. 


\section{LA GERENCIA DEL MARKETING EDUCATIVO EN EL CONTEXTO \\ UNIVERSITARIO DEL ESTADO YARACUY}

Hamlet Trejo y Mireya Valdez.

(PP. 4-26)

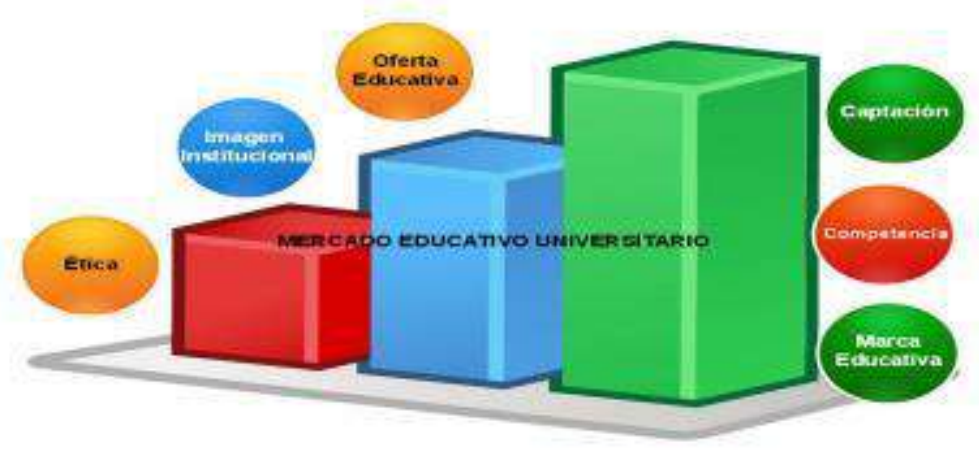

\section{Imagen 1. Categoría Mercado Educativo Universitario y subcategorías}

Imagen Institucional: Este elemento es considerado de gran importancia por los actores sociales al momento de que un potencial cliente, seleccione la institución donde desea cursar sus estudios, así, aunque es deseable tener una imagen positiva, el actor social cinco señala el fenómeno de que hay quien escoge una universidad porque considera que son menos exigentes y obtendrá fácilmente un título universitario.

Oferta Educativa: Los actores sociales estiman que la oferta debe caracterizarse por su variedad y amplitud. Por su parte el actor social nueve, gerente de la UPTYAB, revela que en las instituciones públicas debido a la demanda excesiva de ciertas carreras para la que no existe capacidad, se trata de canalizar la oferta hacia otras carreras similares.

Captación: Los actores sociales siete y ocho, gerentes del CUAM y del IUTAJS respectivamente, develan algunas de las estrategias empleadas para la captación de estudiantes, así el CUAM utiliza el acercamiento que hacen los estudiantes del servicio comunitario, para dar a conocer las especialidades de la institución y se ubican en zonas adyacentes a los centros de educación universitaria para repartir material informativo. Ahora bien, la gerente del IUTAJS, señala las charlas, películas, testimonios llevadas a los liceos y la entrega de material P.O.P (objetos con el logo de la institución) en sitios claves de la ciudad.

Competencia: Las instituciones públicas compiten con las privadas, con la intención de acaparar la mayor parte del mercado educativo universitario, es decir se parte de la idea de que toda persona que quiera estudiar en una universidad pública pueda hacerlo, tal como lo 


\section{LA GERENCIA DEL MARKETING EDUCATIVO EN EL CONTEXTO \\ UNIVERSITARIO DEL ESTADO YARACUY}

Hamlet Trejo y Mireya Valdez.

(PP. 4-26)

aportan los actores sociales seis y nueve, docente y gerente de la UPTYAB, respectivamente. De igual forma destaca, la utilización de la publicidad, con sus diferentes técnicas para sobresalir frente a la competencia, tal como se patentiza en los discursos de los actores sociales, tres, seis y ocho.

Ética: Sobre este aspecto, los actores sociales siete y ocho, gerentes del CUAM y IUTAJS, respectivamente, destacan la transparencia que debe caracterizar a la oferta académica, mientras que el actor social nueve, gerente de la UPTYAB, destaca los principios que deben guiar al proceso de educativo.

Marca Educativa: Los actores sociales, siete y ocho, gerentes del CUAM y el IUTAJS respectivamente, ven en la identificación de sus organizaciones con el lema corporativo, la característica que los distingue de la competencia, mientras que el actor social nueve, gerente de la UPTYAB, apela a la historia de esta casa de estudio, decana de la educación universitaria en el estado Yaracuy.

Categoría Estudiante Cliente Externo: Esta categoría y las respectivas subcategorías están presentes en la imagen 2

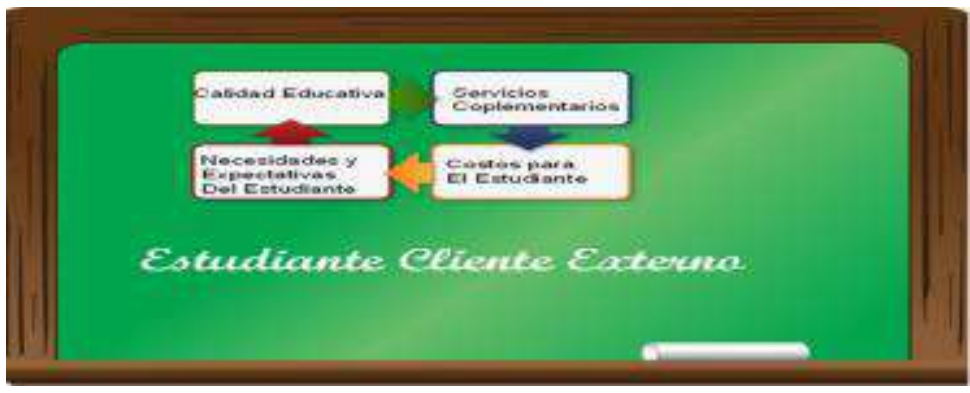

\section{Imagen 2. Categoría Cliente Externo y subcategorías}

Calidad Educativa: Las voces de los actores sociales uno, estudiante del CUAM y seis docente de la UPTYAB, consideran que conocer las expectativas y necesidades de los estudiantes genera calidad. Dentro de este marco, manifiesta el actor social uno, opina que los estudiantes se sienten satisfechos si la institución universitaria les ofrece calidad educativa. Por otra parte, el actor social cinco, educador del IUTAJS, considera que la calidad educativa se refleja en los contenidos programáticos y evaluaciones. Adicionalmente, el actor social seis docente, considera que tanto la dotación de la 


\section{LA GERENCIA DEL MARKETING EDUCATIVO EN EL CONTEXTO \\ UNIVERSITARIO DEL ESTADO YARACUY}

Hamlet Trejo y Mireya Valdez.

(PP. 4-26)

institución como un buen personal son parte de la calidad educativa y estima que cuando existe calidad, hay mayor captación de estudiantes.

Servicios Complementarios: Aparte de la educación formal, las instituciones educativas universitarias deben brindar otros servicios, desde esta perspectiva los actores sociales uno, dos y tres, discentes de las tres organizaciones de este estudio, reseñan los que ellos consideran sobre este aspecto, que necesitan actualmente: Servicios tecnológicos, médicos, bibliotecas. Por su parte el actor social nueve, gerente de la UPTYAB, valora los servicios de comedor y transporte, porque opina que de no existir muchos educandos abandonarían sus estudios.

Necesidades y Expectativas del Estudiante: Dos actores sociales pertenecientes al CUAM, estudiante y docente respectivamente, señalan que la gerencia debe acercarse a los estudiantes para conocer las necesidades y expectativas de los educandos. De acuerdo con los actores sociales dos y tres, estudiantes del IUTAJS y la UPTAB, estiman que la gerencia de sus respectivas instituciones si conoce las necesidades y expectativas de los educandos, pero prefieren ignorarlas. Mientras el profesor del IUTAJS, opina que la gerencia de su institución conoce las expectativas de los estudiantes, el docente de la UPTAB, cree que el conocimiento es poco.

Costos para el Estudiante: El actor social uno, señala que se inscribió en la UPTAB, porque es pública (gratuita) y no debe trasladarse a otro estado. Tanto el estudiante de la UPTAB, como la profesora del CUAM, consideran que el uso de internet en la formación académica como en algunos procedimientos administrativos, disminuyen los costos para los educandos. De igual forma la profesora del CUAM, estima que la matrícula que se cobra en esa casa de estudio es elevada, y sugiere que la gerencia debería bajarla para aumentar la captación. En contraste, la gerente del CUAM señala que los convenios de pago (semestre financiado) alivian la carga económica al estudiante, al tiempo que la gerente del IUTAJS, reconoce como una limitación para atraer estudiantes, los costos de los aranceles por semestre.

\section{Categoría Docente Cliente Interno}

Esta categoría está representada en la imagen 3 


\section{LA GERENCIA DEL MARKETING EDUCATIVO EN EL CONTEXTO \\ UNIVERSITARIO DEL ESTADO YARACUY}

Hamlet Trejo y Mireya Valdez.

(PP. 4-26)

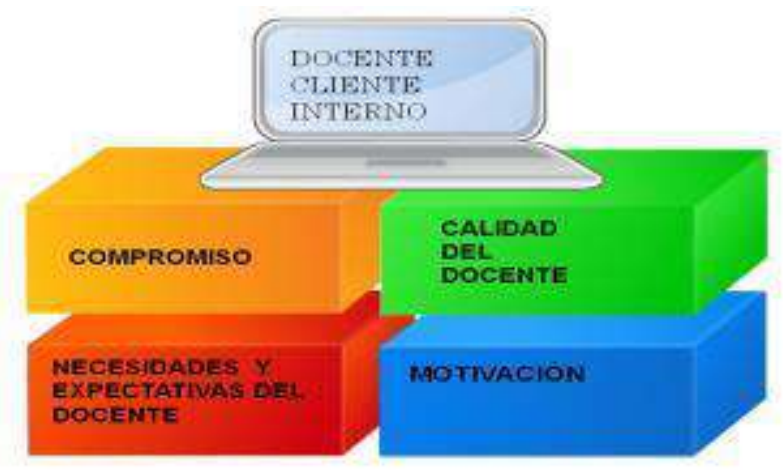

Imagen 3. Categoría Docente Cliente Interno y subcategorías

Compromiso: Para el actor social uno, estudiante del CUAM, un profesor comprometido es exigente, cumple con la planificación. Para el actor social tres, estudiante de la UPTYAB, el docente comprometido, actúa con profesionalismo, entrega y probidad. Para la profesora del CUAM y el profesor de la UPTYAB, un docente comprometido, da lo máximo de sí para transmitir conocimiento. Según, el educador del IUTAJS, manifiesta que un docente comprometido, lucha por la calidad y la formación de profesionales capacitados.

Calidad del Docente: Sobre el tema, el actor social dos, estudiante del IUTAJS, al aumentar la calidad docente, aumenta la captación de una institución universitaria. Para la profesora del CUAM, cuando la institución no facilita cursos de actualización a los docentes, se puede recurrir al aprendizaje cooperativo. De acuerdo con el profesor del IUTAJS, la institución facilita a sus docentes la capacitación en la utilización de la utilización de las Tecnologías de Información y Comunicación. El educador de la UPTYAB, valora la calidad docente en relación con la innovación y la creatividad. Por su parte, la gerente del IUTAJS, la calidad docente precisa del dominio de tecnología, para hacer el hecho educativo más interesante para las nuevas generaciones.

Necesidades y Expectativas del Docente: El actor social cuatro, educadora del CUAM, señala que la gerencia de la institución no se acerca a los docentes para conocer sus necesidades y expectativas, sino que la iniciativa proviene de los profesores. Por su parte el profesor de la UPTYAB, indica que la gerencia de la universidad no conoce las necesidades y expectativas de los docentes y a parte de los salarios son importantes los reconocimientos al trabajo realzado. Indica así mismo, la gerente del IUTAJS, también considera de 


\section{LA GERENCIA DEL MARKETING EDUCATIVO EN EL CONTEXTO \\ UNIVERSITARIO DEL ESTADO YARACUY}

Hamlet Trejo y Mireya Valdez.

(PP. 4-26)

elementos de satisfacción diferentes al dinero: Reconocimientos, trato cordial, oportunidades de crecimiento profesional.

Motivación: De acuerdo con el actor social seis, profesor de la UPTYAB, la gerencia no reconoce o reconoce poco los méritos de los docentes. A todas estas, los gerentes del IUTAJS y de la UPTYAB, comentan sobre las charlas de motivación al personal docente, para que den clases innovadoras o se capaciten para el uso de las herramientas tecnológicas.

\section{Categoría Entorno Institucional}

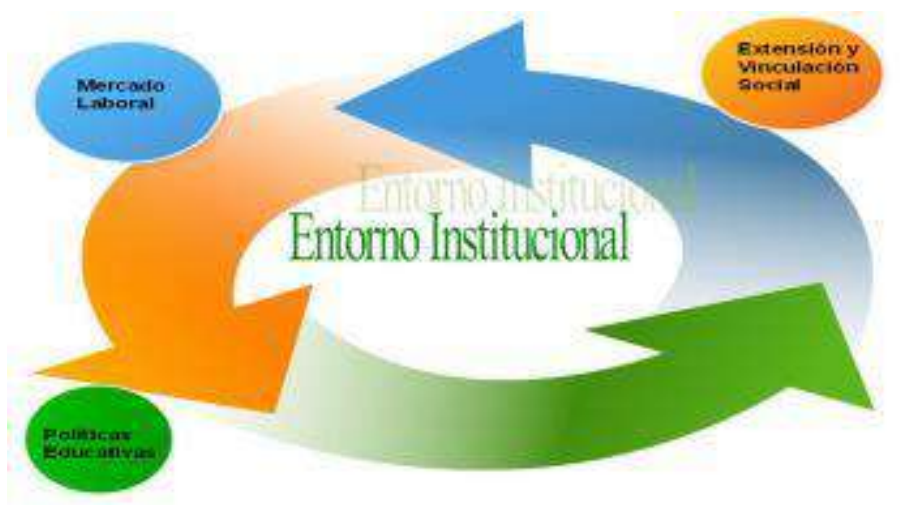

\section{Imagen 4. Categoría Entorno Institucional y subcategorías}

Mercado Laboral: Para el actor social dos estudiante del IUTAJS, las necesidades de la empresa por una profesión en particular la llevaron a estudiar una carrera. Por su parte el actor social siete, gerente universitaria del CUAM, da cuenta de la importancia que tiene conocer el contexto laboral de las especialidades que se dictan en las instituciones universitarias. Adicionalmente, el actor social siete, gerente del IUTAJS, destaca que dentro de las empresas existen potenciales estudiantes que requieren formación profesional. Mientras que el gerente del UPTYAB, señala que existe una demanda de profesionales egresados de esa casa de estudios, por parte de las empresas.

Políticas Educativas Para el actor social tres, estudiante de la UPTYAB, las universidades públicas facilitan el ingreso, además señala que en el caso de esta casa de estudios la infraestructura no es acorde a la cantidad de estudiantes. Sobre esta subcategorías, los actores sociales seis y nueve, docente y gerente de la UPTYAB, respectivamente manifiestan que atender las necesidades educativas de los interesados en ingresar al sistema de educación universitario es una política de estado, el gerente de la

Volumen $21 \quad N^{\circ} 2$ Mayo-Agosto 2017. ISSN: 2244-7296 


\section{LA GERENCIA DEL MARKETING EDUCATIVO EN EL CONTEXTO \\ UNIVERSITARIO DEL ESTADO YARACUY}

Hamlet Trejo y Mireya Valdez.

(PP. 4-26)

UPTAB, explica como la Oficina de Planificación del Sector Universitario (OPSU) actúa como un organismo regulador de la oferta académica.

Extensión y Vinculación Social: De acuerdo con los planteamientos de los actores sociales seis, siete y nueve, educador de la UPTYAB, gerente del IUTAJS y de la UPTYAB, respectivamente, las instituciones educativas tienen el deber de prestar servicio a las comunidades en la solución de problemas puntuales, asesorar en asuntos de trámites ante los entes gubernamentales, así como también servir de enlace entre estos organismos y los ciudadanos. Además, el actor social siete, gerente del CUAM, devela que el servicio comunitario, ha representado para la institución una oportunidad para promocionar su oferta académica

\section{Categoría Sistema Organizacional}

Esta categoría y sus subcategorías están aparecen en la imagen 5

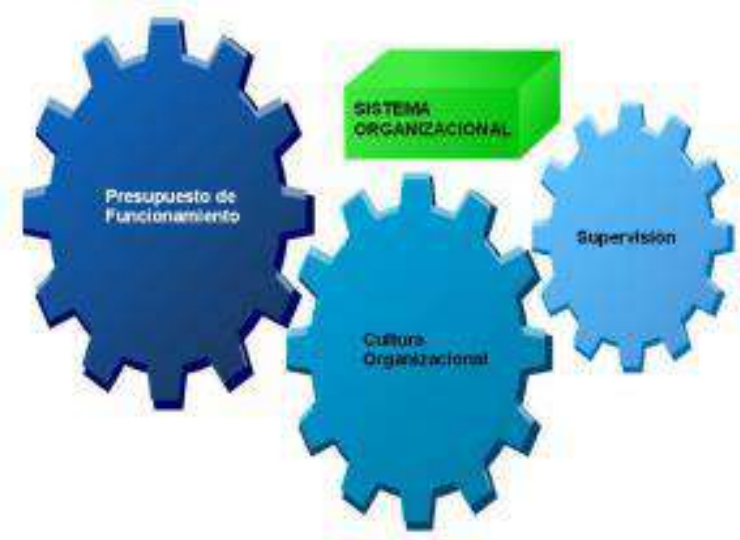

\section{Imagen 5. Categoría Sistema Organizacional y Subcategorías}

Supervisión: De acuerdo con el actor social dos, estudiante del IUTAJS, existen profesores con conocimientos pero sin pedagogía. Según los profesores del CUAM y del IUTAJS, la supervisión es necesaria para el aseguramiento de la calidad. Para el actor social nueve, gerente de la UPTYAB, se debe presionar para que el personal docente se cumpla con las reglas de la institución

Cultura Organizacional: Reseña el actor social cinco, profesor del IUTAJS, existen normas no escritas en la organización que responsabilizan a los docentes cuando la cifra de estudiantes aprobados en una asignatura está por debajo de ciertos límites. El docente de la 


\section{LA GERENCIA DEL MARKETING EDUCATIVO EN EL CONTEXTO \\ UNIVERSITARIO DEL ESTADO YARACUY}

Hamlet Trejo y Mireya Valdez.

(PP. 4-26)

UPTYAB, por su parte señala que la gerencia de esa institución promueve el uso de las TICS por parte de los profesores, pero las palabras no están respaldadas por acciones efectivas. Adicionalmente, el gerente de la UPTYAB, indica que en las instituciones educativas tanto privadas como públicas, existen reglamentos que el personal docente incumple.

Presupuesto de Funcionamiento: Devela el actor social siete, gerente del CUAM que la gerencia debería tomar en cuenta los costos para el estudiante, pero priva el hecho de ser una organización lucrativa. La gerente del IUTAJS, manifiesta sobre los costos actuales para las instituciones universitarias privadas, que resultan difíciles de cubrir con sólo el aporte económico de los estudiantes, por lo que se precisan nuevas alternativas de ingresos. El gerente de la UPTYAB, muestra el panorama de la universidad pública, que proporciona educación gratuita con los aportes del Estado para su funcionamiento, pero que resultan insuficientes para cubrir la gran cantidad de estudiantes que deben atender.

\section{CONSIDERACIONES Y REFLEXIONES FINALES}

Sobre el mercado educativo universitario resulta interesante, que tal como lo señalan los textos de economía se trata de un mercado competitivo, porque existen numerosas instituciones que realizan ofertas académicas y gran cantidad de personas que demandan servicios educativos, que tiende a crecer cada año, dadas las características demográficas venezolanas y las políticas de Estado. Sobre este particular se destaca que a pesar de que las IEU públicas valiéndose de su ventaja competitiva (gratuidad del servicio), acaparan la mayor porción del mercado, el hecho de que existan las IEU privadas da cuenta de que es una actividad rentable.

Adicionalmente, el Estado a través de las Universidades Politécnicas Territoriales, diseña la oferta de acuerdo a lo que estima son las carreras que necesita la región, tal concepción no necesariamente coincide con las expectativas del estudiante, éste por lo tanto buscará en las IEU privadas esa formación que las organizaciones públicas no le ofrecen. Esta característica invita a una discusión: ¿Una persona debería poder estudiar cualquier profesión que desee, independientemente de que no exista la oportunidad de emplearse en 


\section{LA GERENCIA DEL MARKETING EDUCATIVO EN EL CONTEXTO \\ UNIVERSITARIO DEL ESTADO YARACUY}

Hamlet Trejo y Mireya Valdez.

(PP. 4-26)

esa área, por lo que la oferta de las carreras no deberían ser una imposición por parte del Estado? Es de esperar que se incentive el estudio de ciertas profesiones de poca demanda pero necesarias, no obstante la elección del programa a cursar, siempre debe partir del cliente.

Por otra parte, los servicios complementarios, no sólo son importantes para hacer más atractiva la oferta, sino que además son necesarios para que el educando permanezca en la institución, es decir, lograr la fidelización del cliente. Aunque en las IEU públicas en su mayoría estos servicios son gratuitos o de bajo costos, resulta revelador un hallazgo de la investigación en relación a tener un transporte nocturno: Los estudiantes estarían dispuesto a pagarlo.

La anterior declaración se valora como una oportunidad de mejorar la oferta académica de las IEU privadas, ya que en caso de que no puedan prestar el servicio directamente porque les resulte costoso, la organización podrá firmar algún tipo de convenio o alianza con otra que brinde el servicio. Seguramente, una investigación sobre este tema, podría revelar sobre otros servicios complementarios que no brindan estas instituciones y que por estimarlos necesarios, los clientes consentirían en sufragarlos de forma adicional a lo que les corresponde por matrícula o mensualidad.

En el caso del educador universitario, este posee una serie de necesidades que se corresponden con la teoría de Maslow (1991), así se tienen las siguientes: Básicas (Salario), de Seguridad (Salud laboral), de Estima (Amistad con colegas y otros integrantes de la organización), de Reconocimiento (Valoración de sus méritos) y Autorrealización (Amor por la profesión). De igual forma se interpreta que las teorías de McClelland (1989), dan cuenta de la motivación al logro (énfasis en los resultados) o motivación a la afiliación (necesidad de interacción social), y la de Vroom (1995) la cual trata sobre las expectativas que proporciona alcanzar un resultado y las consecuencias que provoca. Conviene por lo tanto, realizar investigaciones en este campo que permitan a la gerencia universitaria, conocer cómo motivar a su cuerpo profesoral, además de teorías adicionales como la de los reforzamientos o la del establecimiento de metas.

Sobre la categoría entorno institucional, se destaca que las organizaciones del mercado laboral y las IEU, deben estar en contacto permanente o establecer una relación simbiótica, 


\section{LA GERENCIA DEL MARKETING EDUCATIVO EN EL CONTEXTO \\ UNIVERSITARIO DEL ESTADO YARACUY \\ Hamlet Trejo y Mireya Valdez. \\ (PP. 4-26)}

de modo tal que egresen al campo de trabajo los profesionales con las competencias que este requiere y no se siga graduando a desempleados.

Para finalizar, dentro dela categoría de los sistemas organizacionales llama la atención la supervisión pedagógica por el hecho de estar presente principalmente en el discurso de docentes, por lo que se infiere que están de acuerdo con este proceso, ya que permite asegurar la calidad educativa, aunque, realizada de modo tal que el educador se sienta respetado (con énfasis en la comunicación horizontal, es decir, no se establecen jerarquías) y que se está contribuyendo con su crecimiento profesional.

\section{REFERENCIAS}

Bustos, H. (2010). Sobre marketing educacional. Libro Digital. Santiago, Chile: Bucket Model. Business Education.

Didriksson, A. (2005). De la reforma a la innovación: La Universidad de nuevo necesaria. Cuaderno de Investigación en la Educación. Número 20, diciembre de 2005. [Artículo en línea] Disponible

http://cie.uprrp.edu/cuaderno/ediciones/19/pdfcuaderno19/c19art1.pdf $\quad$ [Consulta: 2014, Marzo 02].

Heidegger, M. (1999). Ontología. Hermenéutica de la Facticidad. Madrid: Alianza

Knight, J. (2005). Un modelo de internacionalización: Respuestas a nuevas realidades y retos. En Educación Superior en América Latina. La Dimensión Internacional. (Pp. 138). Bogotá: Mayol Ediciones.

Malpica, M. y Rossel, R. (2010). Gerencia Universitaria: Tiempo de Reflexión. Revista Ciencias de la Educación. Vol. 20. Núm. 35. Pp. 185-203. Enero-Junio 2010.

Manes, J. (2008). Marketing para instituciones educativas. (2da Ed.) Buenos Aires: Granica.

Maslow, A. (1991). Motivación y Personalidad. Madrid: Díaz de Santos. 


\section{LA GERENCIA DEL MARKETING EDUCATIVO EN EL CONTEXTO \\ UNIVERSITARIO DEL ESTADO YARACUY \\ Hamlet Trejo y Mireya Valdez. \\ (PP. 4-26)}

McClelland, D. (1989). Estudio de la Motivación Humana. Madrid: Narcea.

Misión Alma Mater (2009). Educación Universitaria y Socialista. [Documento en línea] Disponible http://www.unearte.edu.ve/media/informacion/pdf/mision_alma_mater.pdf [Consulta: 2014, Marzo 02].

Muro, X. y Picón, G. (2005). La Gerencia Universitaria en Venezuela: Un estudio crítico interpretativo de su discurso. Revista Informe de Investigaciones Educativas. Vol. XIX. Núm. 1. Pp. 13-36.

Pérez, L. (2004). Marketing Social: Teoría y Práctica. México: Paerson Prentice Hall.

Rivero, E. y Goyo, A. (2012). La Gerencia Universitaria Venezolana ante los Nuevos Retos de la Sociedad del Siglo XXI. Revista Gestión y Gerencia. Vol. 6. Núm. 2. Pp. 425. Mayo-Agosto 2012.

Sainz, J. (2010). El Plan de Marketing en la PYME. (2da Ed). Madrid: ESIC.

Saldaña, J. (2013). Mercadotecnia para instituciones educativas. Revista electrónica Contaduría y Administración. Núm. 192. Octubre 2013. Universidad Nacional Autónoma de México. Disponible en http://contaduriayadministracionunam.mx/articulo-14-493-79.html [Consulta: 2014, Marzo: 06].

Sandín, M. (2003). Investigación Cualitativa en Educación. Fundamentos y Tradiciones. Madrid: Mc Graw Hill.

Taplero, E. y López, L. (2006). Gestión Escolar Inteligente. Instituciones Educativas Neosistémicas del siglo XXI. Florencia, Colombia: Universidad de La Amazonia.

Vroom, V. (1995). Work and Motivation.San Francisco, California: Jossey-Bass. 